\title{
Investigating photoresponsivity of graphene-silver hybrid nanomaterials in the ultraviolet
}

Cite as: J. Chem. Phys. 152, 044709 (2020); https://doi.org/10.1063/1.5138722

Submitted: 19 November 2019 . Accepted: 29 December 2019 . Published Online: 27 January 2020

Preeti Deshpande (D), Priyanka Suri, Hyeon-Ho Jeong, Peer Fischer, Arindam Chosh, and Ambarish Chosh (D)

\section{COLLECTIONS}

Paper published as part of the special topic on Emerging Directions in Plasmonics

Note: This paper is part of the JCP Special Topic on Emerging Directions in Plasmonics.
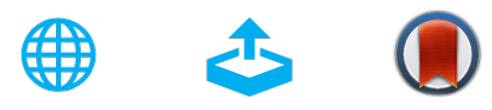

\section{ARTICLES YOU MAY BE INTERESTED IN}

Gold nanoparticle on semiconductor quantum dot: Do surface ligands influence Fermi level equilibration

The Journal of Chemical Physics 152, 044710 (2020); https://doi.org/10.1063/1.5138216

Long-lived charge separation in two-dimensional ligand-perovskite heterostructures

The Journal of Chemical Physics 152, 044711 (2020); https://doi.org/10.1063/1.5131801

A comparative first-principles investigation on the defect chemistry of $\mathrm{TiO}_{2}$ anatase

The Journal of Chemical Physics 152, 044110 (2020); https://doi.org/10.1063/1.5138902

Lock-in Amplifiers

Find out more today

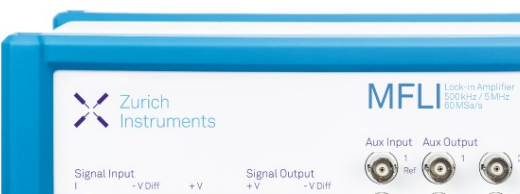

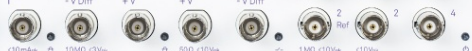

Zurich

- Instruments 


\title{
Investigating photoresponsivity of graphene-silver hybrid nanomaterials in the ultraviolet
}

\author{
Cite as: J. Chem. Phys. 152, 044709 (2020); doi: 10.1063/1.5138722 \\ Submitted: 19 November 2019 - Accepted: 29 December 2019 • \\ Published Online: 27 January 2020
}

\author{
Preeti Deshpande, ${ }^{1}$ (D) Priyanka Suri, ${ }^{1}$ Hyeon-Ho Jeong, ${ }^{2}$ Peer Fischer, ${ }^{2,3}$ Arindam Ghosh, \\ and Ambarish Ghosh
}

\begin{abstract}
AFFILIATIONS
${ }^{1}$ Centre for Nano Science and Engineering, Indian Institute of Science, Bangalore, India

${ }^{2}$ Max Planck Institute for Intelligent Systems, Heisenbergstr. 3, 70569 Stuttgart, Germany

${ }^{3}$ Institute for Physical Chemistry, University of Stuttgart, Pfaffenwaldring 55, 70569 Stuttgart, Germany

${ }^{4}$ Department of Physics, Indian Institute of Science, Bangalore, India
\end{abstract}

Note: This paper is part of the JCP Special Topic on Emerging Directions in Plasmonics.

a) Author to whom correspondence should be addressed: ambarish@iisc.ac.in

\begin{abstract}
There have been several reports of plasmonically enhanced graphene photodetectors in the visible and the near infrared regime but rarely in the ultraviolet. In a previous work, we have reported that a graphene-silver hybrid structure shows a high photoresponsivity of $13 \mathrm{~A} / \mathrm{W}$ at $270 \mathrm{~nm}$. Here, we consider the likely mechanisms that underlie this strong photoresponse. We investigate the role of the plasmonic layer and examine the response using silver and gold nanoparticles of similar dimensions and spatial arrangement. The effect on local doping, strain, and absorption properties of the hybrid is also probed by photocurrent measurements and Raman and UV-visible spectroscopy. We find that the local doping from the silver nanoparticles is stronger than that from gold and correlates with a measured photosensitivity that is larger in devices with a higher contact area between the plasmonic nanomaterials and the graphene layer.
\end{abstract}

Published under license by AIP Publishing. https://doi.org/10.1063/1.5138722

\section{INTRODUCTION}

The spectrally flat, low intrinsic absorption of $2.3 \%$ in graphene $^{1}$ is large considering it is only an atom thick; however, it still is a limiting factor for optoelectronics, such as photodetection applications. Various schemes to enhance the interaction of light with graphene ${ }^{2-6}$ have been implemented, of which integration with plasmonic nanoparticles is particularly attractive. ${ }^{7-10}$ The subwavelength localization and enhancement of the electric field around the nanoparticles can lead to a higher responsivity at the plasmon resonance. They also offer tunability in terms of spectral response, e.g., by changing the shape, size, and material, or polarizationresponse, e.g., by changing the aspect ratio. ${ }^{11}$ By engineering the coupling between the plasmonic nanoparticles, ${ }^{12,31}$ e.g., sandwiching the graphene between two plasmonic nanospheres, ${ }^{13}$ extremely sensitive photodetectors have been developed, most of which operate in the visible to near IR wavelengths. So far, gold has been used most extensively in the literature due to its stable and nonoxidizing nature. ${ }^{12,14,15}$ However, the plasmon resonance for gold lies mostly in the visible and near IR depending on the geometry and size of the nanoparticle. On the contrary, silver supports plasmon resonance in the near-UV range making it a better plasmonic material for enhanced photodetection in the UV.

In our previous work, ${ }^{16}$ we have reported a hybrid graphene photodetector by integrating CVD graphene with a large area hexagonally patterned array of silver nanoparticles. The device shows high responsivity across the UV range with two maxima of $3.2 \mathrm{~A} / \mathrm{W}$ at the wavelength of $\lambda=370 \mathrm{~nm}$ and $14.5 \mathrm{~A} / \mathrm{W}$ at $\lambda=280 \mathrm{~nm}$. The plasmonic near field enhancement due to silver nanoparticles could easily explain the high responsivity at $\lambda=370 \mathrm{~nm}$. The anomalous response at $\lambda=280 \mathrm{~nm}$, however, has not been explained and was assumed to be related to the enhanced density of states of graphene (Van Hove singularity) around this wavelength $(\sim 250 \mathrm{~nm})$. There could be a cumulative effect of multiple factors such as enhanced absorption and effective carrier separation near the singularity aided by the strong disorder in the graphene due to the transfer process. 
There could be higher injection of photocarriers due to hot electrons as well. Here, we explore some of these possibilities and investigate the role of the plasmonic material and the geometrical properties such as size and spatial arrangement in the anomalous response at $270 \mathrm{~nm}$. We have made devices with gold or with silver nanoparticles of similar dimensions, and we also use silver nanoparticles of different sizes and spatial order. We first probe the optoelectronic response of three devices using a photocurrent measurement setup and study their responsivities in the $250-560 \mathrm{~nm}$ wavelength range. The optical response of the plasmonic substrates is modeled using COMSOL and verified using UV-visible (UV-vis) optical extinction spectroscopy. Finally, the role of local doping and strain due to plasmonic nanoparticles is probed using Raman scattering spectroscopy.

\section{RESULTS AND DISCUSSIONS}

Figure 1 shows the schematic of graphene-plasmonic particle hybrid photodetectors and a representative scanning electron microscope (SEM) image of one of the hybrid geometries used in the experiments reported here. We compare results from 3 types of hybrids (details mentioned in Table I). The particle diameters are approximately $60 \mathrm{~nm}$ for silver and $50 \mathrm{~nm}$ for gold with a center to center spacing of $200 \mathrm{~nm}$ and $110 \mathrm{~nm}$ for devices named "AG 60 " and "AU 50," respectively. They are fabricated using a combination of block copolymer micellar lithography (BCML) and glancing angle deposition (GLAD) ${ }^{17}$ (see the supplementary material for SEM images). The third substrate, named "AG 120," has silver nanoparticles of $120 \mathrm{~nm}$ diameter fabricated by nanoimprint lithography. More details on device fabrication are available in the Device fabrication subsection in the Experimental Section. Commercially available CVD grown single layer graphene from $\log 9$ Materials is transferred onto plasmonic substrates using the standard PMMA based wet transfer method. For electrical characterization, contacts are fabricated by placing a stencil mask and then evaporating $5 \mathrm{~nm}$ chromium and $60 \mathrm{~nm}$ gold in a thermal evaporator. Contacts are then made to the device by mounting it on the printed circuit board (PCB) and using epoxy bonding. The IV characteristics were found to be linear in the range of $\pm 150 \mathrm{mV}$ of the source-drain voltage. The effective two probe resistance of the devices in dark (nonilluminated conditions) and vacuum is $362 \Omega, 277 \Omega$, and $280 \Omega$ for the AG 60, AU 50, and AG 120 devices, respectively. Gate dependent device resistance was probed to find the doping of graphene (see Fig. S2 for resistance vs gate voltage measurement for AG 60);

(a)

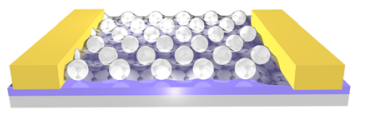

(b)

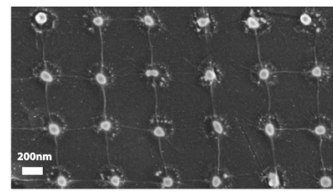

FIG. 1. Graphene-nanoparticle hybrids. (a) Schematic of the hybrid device. The base nanoparticle layer consists of an array of silver or gold nanoparticles. A CVDgrown single layer graphene is transferred onto plasmonic substrates using the wet transfer method. Source drain electrodes are fabricated by depositing $5 \mathrm{~nm}$ chromium and $60 \mathrm{~nm}$ gold through a prepatterned stencil mask. (b) SEM image of the silver-graphene hybrid (AG 120) with a particle diameter of 120 nm.
TABLE I. Geometrical and material properties of the three graphene-plasmonic hybrid devices.

\begin{tabular}{lccc}
\hline \hline Device & Material & Diameter $(\mathrm{nm})$ & Spacing $(\mathrm{nm})$ \\
\hline AG 60 & $\mathrm{Ag}$ & 60 & 200 \\
AG 120 & $\mathrm{Ag}$ & 120 & 600 \\
AU 50 & $\mathrm{Au}$ & 50 & 110 \\
\hline \hline
\end{tabular}

however, polymer transfer processes used for large area CVD graphene, coupled with the local doping from nanoparticles, made it difficult to perform these measurements for all the three devices.

For the photoresponse measurements, the devices were flood illuminated under a monochromatic light while the source was switched on and off repeatedly. Then, the photoswitching responses of the devices were obtained by modulating the light on time scales of $10 \mathrm{~s}$ (AG 60), $3 \mathrm{~s}$ (AU 50), and $3 \mathrm{~s}$ (AG 120) corresponding to the changes in current flowing through each device. For the data shown in Fig. 2, we used source-drain voltages $\left(\mathrm{V}_{\mathrm{sd}}\right)$ of $0.35 \mathrm{~V}$ for AG 60 and AU 50 devices and $0.2 \mathrm{~V}$ for the AG 120 device. The devices were subjected to an acclimatization process where the incident light was kept on for a brief period followed by few on-off cycles before the final photoresponse was recorded. All the devices showed positive photoresponse that is the current increased upon illumination. Typical on-off responses with associated exponential fittings at $270 \mathrm{~nm}$ for AG 60 and at $530 \mathrm{~nm}$ for AU 50 hybrid are shown in Figs. 2(a) and 2(b). Their corresponding saturated photocurrent and rise times are extracted from the fitting data.

We further characterize the device response as a function of wavelength [see Fig. 2(c)] for all the three devices. The responsivities of AG 60 and AG 120 showed two maxima: the first $1.98 \mathrm{~A} / \mathrm{W}$ at $\lambda=370 \mathrm{~nm}(\mathrm{AG} \mathrm{60)}$ ) and $0.15 \mathrm{~A} / \mathrm{W}$ at $\lambda=380 \mathrm{~nm}$ (AG 120) and the second $82 \mathrm{~A} / \mathrm{W}$ (AG 60) and $1.25 \mathrm{~A} / \mathrm{W}$ (AG 120) at $\lambda=270 \mathrm{~nm}$ for both devices. The AU 50 device has a maximum photoresponsivity of $0.08 \mathrm{~A} / \mathrm{W}$ at $\lambda=510 \mathrm{~nm}$ and shows a photoresponse only up to $460 \mathrm{~nm}$, below which we could only observe light activated drift and no clear sign of on-off photoresponse. The maxima at $\lambda=370 \mathrm{~nm}, 380 \mathrm{~nm}$, and $510 \mathrm{~nm}$ for the three devices correspond to the plasmonic resonances of the underlying nanoparticle array, but the peak at $\lambda=270 \mathrm{~nm}$ for the two silver devices could not be attributed to any plasmonic effects. This will be shown clearly later in Fig. 3(a). We further note that the plasmonic maxima shown by the silver-graphene hybrids are of similar spectral characteristics but slightly higher magnitude compared to those of another Ag-graphene device that we had reported previously. ${ }^{16}$

More crucially, the enhanced responsivity at $\lambda=270 \mathrm{~nm}$ is in both the silver-graphene hybrids (AG 60 and AG 120) but is not seen in the gold-graphene hybrid (AU 50). This suggests the importance of silver as a material to achieve enhanced sensitivity and rules out the possibility that the anomalously large photoresponsivity is primarily due to strain caused by transferring CVD graphene on nanoparticle arrays. The inverse dependence of the rise time on incident power, as shown in Fig. 2(d), suggests the photoresponse process to be photovoltaic in origin. The time scales match with similar 
(a)

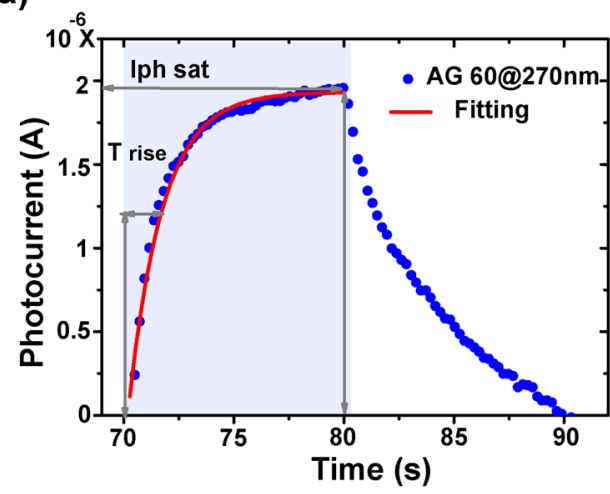

(b)

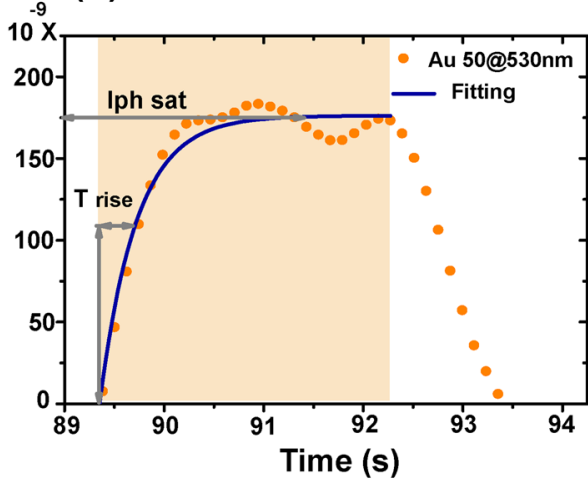

(c)
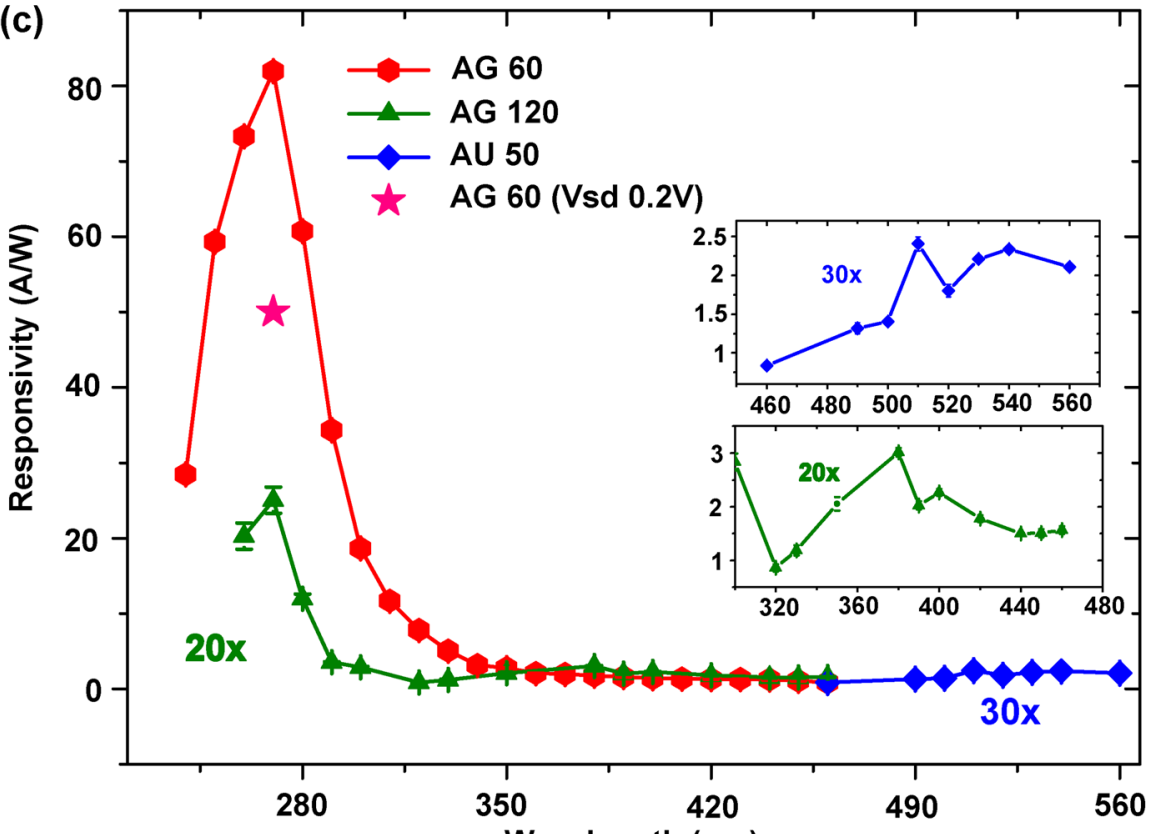

Power on device (nW)

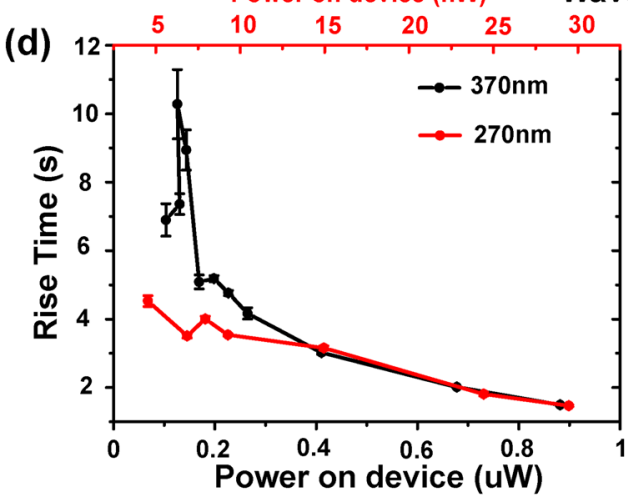

(e)

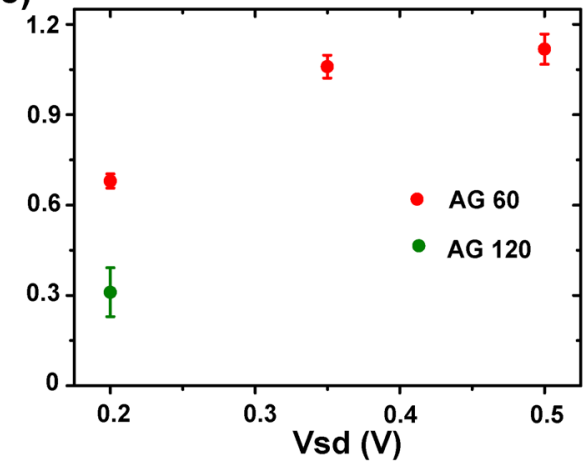

FIG. 2. Typical on-off photoswitching responses of (a) AG 60 at $\lambda=270 \mathrm{~nm}$ and (b) $A U 50$ at $\lambda=530 \mathrm{~nm}$. The solid lines indicate exponential fittings to the data to extract the associated saturated photocurrent (Iph sat) and the rise times $\left(T_{\text {rise }}\right)$. (c) Photoelectric responsivities of three graphene-nanoparticle hybrid devices, AG $60\left(\mathrm{~V}_{\mathrm{sd}}=0.35 \mathrm{~V}\right)$ AG $120\left(V_{\text {sd }}=0.2 \mathrm{~V}\right)$, and $A U 50\left(V_{s d}\right.$ $=0.35 \mathrm{~V}$ ). Insets show the peak responsivities at the plasmonic resonances of AG 120 (green) and AU 50 (blue). The peak responsivity occurs in the deep UV region at $270 \mathrm{~nm}$ for both the $\mathrm{Ag}$ graphene hybrid devices; however, AU 50 does not show any response beyond $460 \mathrm{~nm}$. The highest responsivity is shown by the AG 60 device. (d) Power dependent rise times of $A G 60$ at $V_{s d}$ $=0.35 \mathrm{~V}$ for two different wavelengths $270 \mathrm{~nm}$ and $370 \mathrm{~nm}$. (e) Calculated rise times of $A G 60$ and $A G 120$ at different source-drain voltages $\left(V_{s d}\right)$ measurements in related systems and originate in the large number of trap states present in the CVD grown graphene, as well as the silica surface in contact with the graphene layer. ${ }^{18}$ The large noise in the response time measurements for $370 \mathrm{~nm}$ is related to the low photocurrent and corresponding fitting error. We find similar response times in the other silver device, AG 120, as shown in the measurements of rise time as a function of $\mathrm{V}_{\mathrm{sd}}=0.2 \mathrm{~V}$ at $\lambda=270 \mathrm{~nm}$ [see Fig. 2(e)]. 
(a)

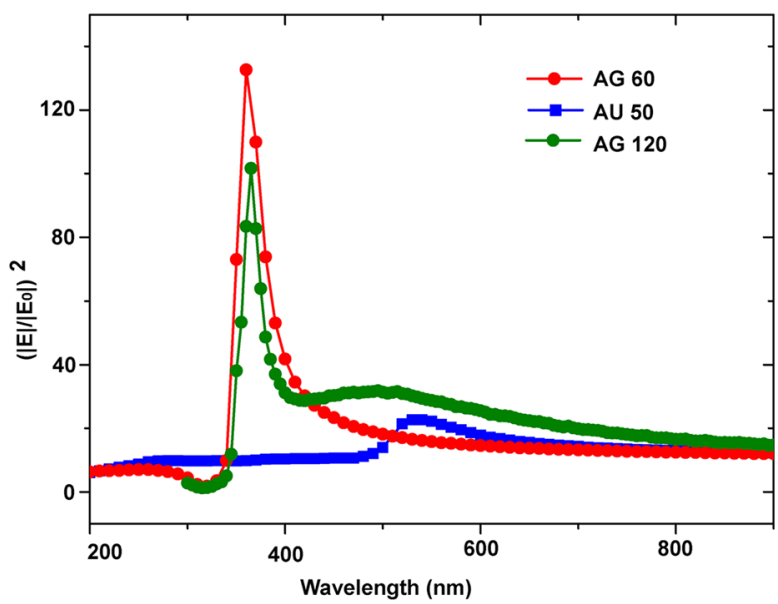

(b)

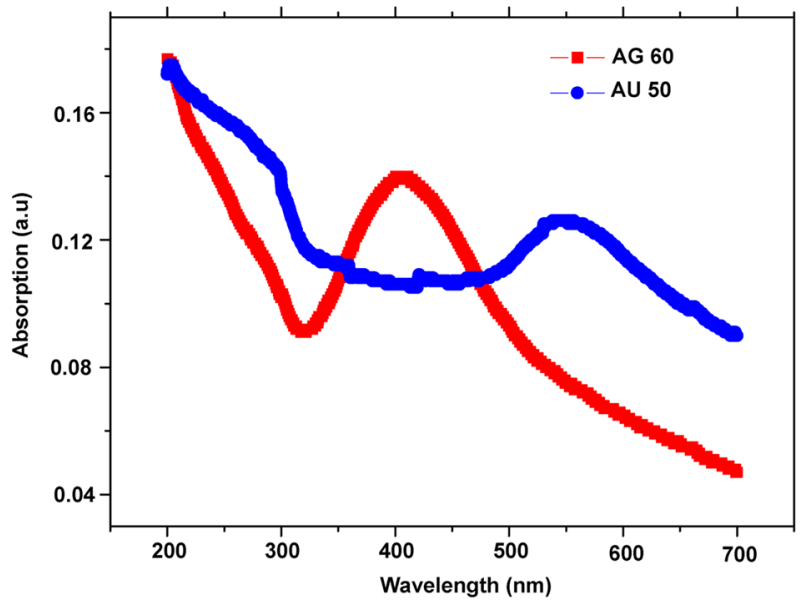

FIG. 3. (a) Simulated total enhancement $\left(|E| /\left|E_{0}\right|\right)^{2}$ max of Ag-graphene and Au-graphene hybrid devices as a function of wavelength. (b) Measured absorption spectra of $A G$ 60 and $A \cup 50$ nanoparticles on a quartz substrate as a function of wavelength.

To investigate the maxima in spectral responsivity, we characterize the optical properties of the plasmonic substrates. We numerically model the geometry [Fig. 3(a)] using COMSOL, a commercially available finite element modeling software. The modeled geometry consists of $\mathrm{Ag}$ and $\mathrm{Au}$ nanoparticles of $50 \mathrm{~nm}$ diameter and $100 \mathrm{~nm}$ lattice spacing for AG 60 and AU 50, respectively, and a single Ag nanoparticle of $120 \mathrm{~nm}$ diameter for AG 120. Details regarding mesh size and boundary conditions used in the simulations are given in the section titled "Experimental section." We model the total enhancement $\left(|\mathrm{E}| /\left|\mathrm{E}_{0}\right|\right)^{2}{ }_{\text {max }}$ that refers to the squared ratio of magnitude of the local to incident electric field over the total volume of the unit cell. The total enhancement as a function of wavelength is plotted in Fig. 3(a). We observe pronounced peaks at $370 \mathrm{~nm}$ for AG 60 and AG 120 and at $530 \mathrm{~nm}$ for AU 50, which correspond to the electric dipole modes of the respective plasmonic geometries.

To experimentally verify the optical properties, we fabricate the plasmonic array on a transparent substrate, a quartz wafer in this case, and characterize it using UV-visible absorption spectroscopy. The measured absorption spectra of AG 60 and AU 50 are shown in Fig. 3(b). The absorption peak of AG 60 lies around $400 \mathrm{~nm}$ and of AU 50 lies around $550 \mathrm{~nm}$, in agreement with the estimates of the simulated resonance wavelengths. The slight disparity between the simulated and UV-vis measurements could occur because substrate effects were not taken into account during simulation. Indeed, the photoresponsivities of the device are also enhanced at slightly shifted wavelengths ( $370 \mathrm{~nm}$ for AG $60 \mathrm{~nm}$ and $510 \mathrm{~nm}$ for AU 50) and clearly can be attributed to plasmonic near field effects.

Clearly, occurrence of the prominent peak at $\lambda=270 \mathrm{~nm}$ only in the Ag-graphene hybrid devices cannot be explained by plasmonic near-field contributions. As reported earlier, the enhanced responsivity at $270 \mathrm{~nm}$ is probably related to the enhanced density of states at the Van Hove singularity in graphene. ${ }^{19-21}$ The induced disorder coupled with multiple scattering centers and large local doping could be important contributing factors for efficient charge carrier extraction. ${ }^{22,23}$ To probe this in detail, we study the effect of local doping and strain, where we measure the surface enhanced Raman signal of the graphene layer and estimate the peak shift, ratio of intensities of $\mathrm{I}_{2 \mathrm{D}} / \mathrm{I}_{\mathrm{G}}$, and ratio of area under the curve $\mathrm{A}_{2 \mathrm{D}} / \mathrm{A}_{\mathrm{G}}{ }^{24}$ of all devices.

Figure 4(a) shows the comparison between Raman spectra of the 3 devices with single layer graphene on the $\mathrm{Si}-\mathrm{SiO}_{2}$ wafer. The defect peak is significantly enhanced in the case of all 3 devices as compared to the single layer graphene. As shown in Fig. S3, the G and $2 \mathrm{D}$ peaks are also enhanced (peak and area under the curve) along with shifts (see Table T1). The wide range of enhancements obtained is due to the nonuniformity of the plasmonic nanoparticle layer which is expected in such large area fabrication techniques. We use the method suggested by Lee et al. ${ }^{25}$ to deconvolute the contributions from strain and doping in Raman spectra of graphenenanoparticle hybrids shown in Fig. 4(b). Figures 4(c) and 4(d) show the change in the position of $\mathrm{G}$ and $2 \mathrm{D}$ peaks of the three devices keeping measurement of the single layer graphene as the reference, with further details given in Table T2 (with the deconvoluted values) of the supplementary material.

We find that graphene is $\mathrm{n}$-doped by Ag and $\mathrm{p}$-doped by $\mathrm{Au}$ (as per the literature), but both the systems are also strained. We obtain a charge carrier density of $6 \times 10^{12} \mathrm{~cm}^{-2}$ (n doping) for the AG 60 device and $3 \times 10^{12} \mathrm{~cm}^{-2}$ ( $\mathrm{p}$ doping) for Au 50 devices, in agreement with the existing literature with CVD graphene. ${ }^{26,27}$ Considering we do not observe any photoresponse with the AU 50 device at $270 \mathrm{~nm}$, we conclude strain due to the transfer process in conjunction with enhanced absorption at the Van Hove singularity cannot play an important role in the enhanced photoresponse of the silver-based devices.

We believe that the enhanced photoresponse of the silver-based devices is related to the graphene being $n$-doped by silver and hot electrons generated in the nanoparticles injected into the graphene. 
(a)

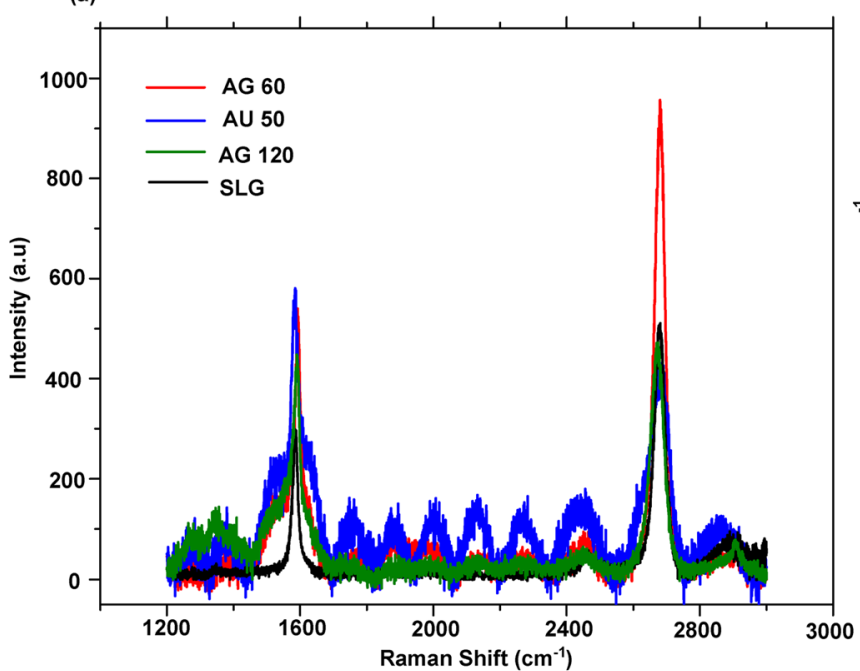

(c)

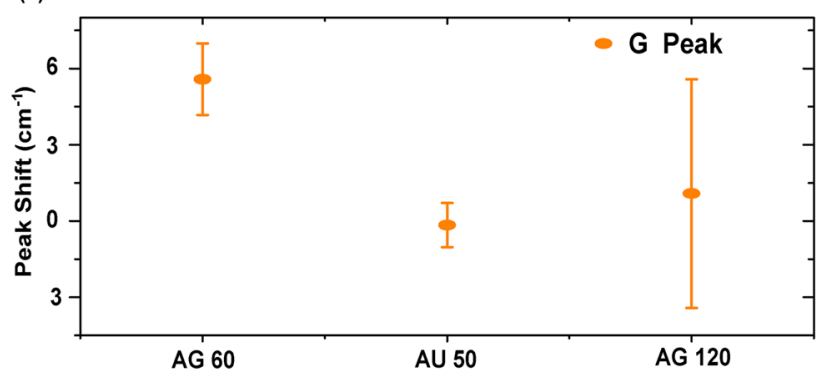

(b)

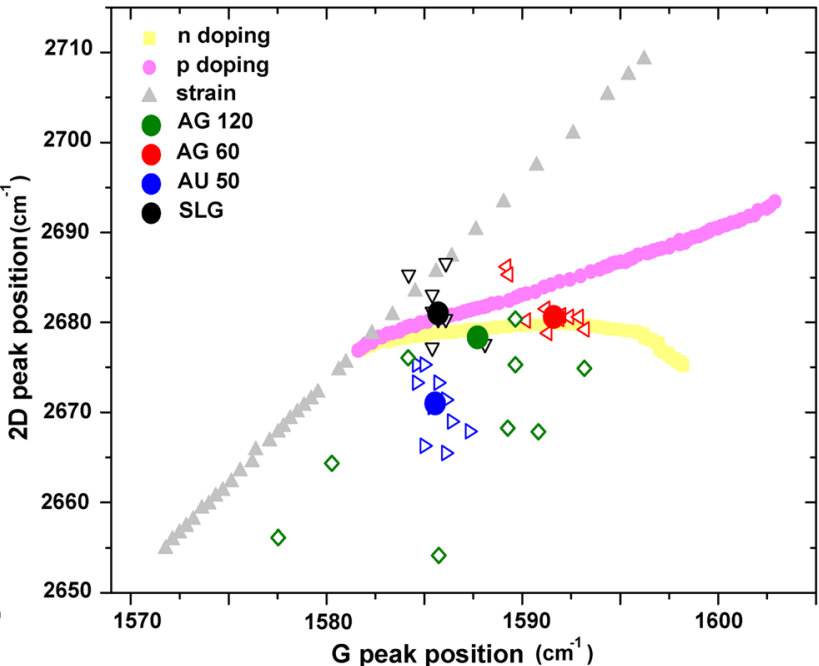

(d)

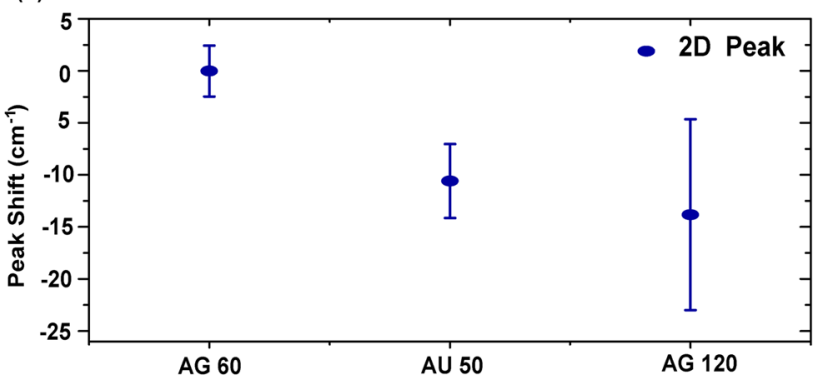

FIG. 4. (a) Raman spectra comparison of $A G 60, A U 50$, and AG 120 with single layer graphene (SLG). The $G$ and $2 D$ peaks are enhanced as compared to SLG. (b) Mapping of $G$ and $2 D$ peaks from all 3 devices and single layer graphene with the literature values for pure strain, $n, p$ doping. The open symbols represent data points taken from various parts on the samples. The same colored filled closed symbols represent the average peak position of the respective devices. Peak shift of the (c) $G$ peak and (d) $2 D$ peak for the 3 devices as compared to a single layer graphene.

The plasmonically induced hot electrons ${ }^{28,29}$ have a higher probability of transmission into the graphene channel compared to the gold-based device (p-doped), in agreement with previous reports. ${ }^{12}$ The effect is expected to be magnified at $\lambda=270 \mathrm{~nm}$, where the density of states of graphene is large due to the Van Hove singularity. Also note the excitation energy at $\lambda=270 \mathrm{~nm}$ is significantly higher than the interband transition energy ( $2.3 \mathrm{eV}$ for gold, $3.7 \mathrm{eV}$ for silver), ${ }^{30}$ which ensures the generation of a significant number of hot electrons. It is also interesting to consider the difference in photoresponse between the two silver devices, where the ratio of responsivities of AG 60 and AG 120 at a source drain voltage of $0.2 \mathrm{~V}$ is nearly 37 [see Fig. 2(c)]. We believe this arises primarily due to the lower area of surface contact between the nanosphere and the graphene device in the AG 120 device as well as different areas of contact between the two devices, with AG 120 containing larger silver nanospheres with a larger spacing. One can also compare with the device described previously, ${ }^{16}$ where the geometries are similar to those of the AG 60 devices, but the maximum responsivity (AG 60 ) was found to be $\sim 8$ times larger at the same source-drain bias as compared to the previous device. This is probably because of a large number of silver nanoparticles (formed due to unshadowed growth during the GLAD step) present in the AG 60 device (see Fig. S1a) compared to the previously reported device, leading to a larger contact area between the silver and the graphene and therefore a larger photoresponse at $\lambda=270 \mathrm{~nm}$.

\section{CONCLUSION}

We have investigated the role of a plasmonic layer of gold and silver nanoparticles for the high UV responsivity in plasmonicgraphene hybrid photodetectors. We characterize the photocurrent, the UV-vis absorption, and the Raman spectra of the hybrids to find the effect of the plasmonic layer (geometry and material) on the local strain and doping. Based on our observations, local doping in Ag-graphene in conjunction with hot electron generation at the wavelength of $270 \mathrm{~nm}$ and the enhanced density of states of graphene around the same energy can result in effective charge carrier separation in the Ag-graphene device as compared to the Augraphene device. Also, the higher surface area of contact between the silver nanoparticles and the graphene layer is found to show 
higher UV photoresponsivity. It will be interesting to investigate the gate dependence and therefore external electrostatic doping effects, although such measurements on large area devices are typically quite challenging.

\section{EXPERIMENTAL SECTION}

\section{Numerical simulations}

We consider silver/gold ${ }^{32}$ nanoparticles of $50 \mathrm{~nm}$ diameter and model the array response by using a periodic boundary condition with lattice size $100 \mathrm{~nm}$. The periodic boundary condition is applied in the $\mathrm{X}$ and $\mathrm{Y}$ directions, and light is incident from the $\mathrm{Z}$ direction at the input port. PMLs (perfectly matched layers) are placed at input and output ports in the $\mathrm{Z}$ direction. A dynamic tetrahedral mesh is used with a minimum mesh size of $0.1 \mathrm{~nm}$ for the particle and maximum size of $\lambda / 6$ for the rest of the geometry. We do not consider the presence of single layer graphene in the current numerical model.

\section{Fabrication details}

Nano-glancing angle deposition (nano-GLAD): An array of $10 \mathrm{~nm} \mathrm{Au}$ nanoparticles with $\sim 110 \mathrm{~nm}$ or $200 \mathrm{~nm}$ center-to-center spacing was patterned using block-copolymer micelle nanolithography (BCML). The block-copolymer micelles were formed by selfassembly in toluene, whose core contains gold salt $\left(\mathrm{HAuCl}_{4}\right)$. Then, they were spin-coated onto a $300 \mathrm{~nm}$ thick $\mathrm{SiO}_{2}$ film grown on a heavily doped p-type Si (100) wafer. This yields a hexagonally ordered monolayer of the micelles on the entire wafer. On treatment with an $\mathrm{H}_{2}-\mathrm{Ar}$ plasma, the Au salts were reduced to form metallic nanoparticles with a $\sim 10 \mathrm{~nm}$ diameter while keeping their original spacings. These act as seeds for subsequent nano-glancing angle deposition (nano-GLAD). Then, Ag or Au nanoparticles were grown on the $\mathrm{Au}$ nanoseeds with the tooling factor of $25 \%$ in a custom-built nano-GLAD system with independent control over the substrate's azimuthal direction and the molecular flux direction. To grow the spherical nanoparticles selectively on the seeds, the flux angle and the azimuthal rotation rates per unit thickness were kept to $87^{\circ}$ and $18^{\circ} / \mathrm{nm}$, respectively, with closed-loop feedback based on measurements of material deposition rates using a quartz crystal monitor (QCM). Although most of the Ag was deposited on the gold seeds, a few smaller spheres in between the larger patterns can also be observed, which were at least 6 times smaller than the Ag nanospheres and therefore of negligible importance to the studies presented here. The typical base pressure and the substrate's temperature during deposition were $1 \times 10^{-6}$ mbar and $\sim 90 \mathrm{~K}$, respectively.

\section{Nanoimprint lithography}

A soft polymer stamp was made using PDMS (polydimethylsiloxane) from commercially available silicon substrates with a hole array of diameter $250 \mathrm{~nm}$ and center to center spacing $600 \mathrm{~nm}$. The soft stamp was imprinted on a photoresist coated substrate (S1805), heated for $2.5 \mathrm{~min}$ at $125^{\circ} \mathrm{C}$, and then taken off leaving the substrate patterned. Substrates were ashed using oxygen plasma to remove the residual photoresist in the hole array. Silver was deposited vertically using thermal evaporation, and lift-off was performed leaving an array of silver discs. The substrates were annealed at $280^{\circ} \mathrm{C}$ in nitrogen ambient to form an array of nanoparticles of $120 \mathrm{~nm}$ diameter.

\section{Device fabrication}

In order to make the silver nanoparticle graphene hybrid device, commercially brought CVD graphene was spin-coated with PMMA $950 \mathrm{k} \mathrm{A} 4$, heated at $120^{\circ} \mathrm{C}$ for $5 \mathrm{~min}$, and put in copper etchant solution ( $30 \mathrm{mg} \mathrm{FeCl}_{3}, 3 \mathrm{ml} \mathrm{HCl}, 30 \mathrm{ml} \mathrm{H}_{2} \mathrm{O}$ ). Once the copper base etches off, the PMMA graphene stack floats on the solution. This stack is then transferred to the DI water bath and rinsed 3 times. Finally, it is scooped up by the BCML substrate and left to air dry overnight. Once air dried, it is dipped in the acetone bath three times for 30 min each and then in the isopropyl alcohol (IPA) bath for $10 \mathrm{~min}$. Following this, it is blow dried by nitrogen.

Contacts were then fabricated by placing a stencil mask and then evaporating $5 \mathrm{~nm}$ chromium and $60 \mathrm{~nm}$ gold in a thermal evaporator. Electrical contacts were then made to the device by mounting it on the PCB and doing epoxy bonding.

\section{Photovoltage characterization}

The devices were mounted in a vacuum chamber with a quartz window pumped down to $10^{-5}$ mbar. We use a Newport arc-Xe lamp and a Newport monochromator, focussing optics to shine one wavelength at a time. Photocurrent measurements are taken by connecting this to Keithley 2450, applying the source drain bias of $0.35 \mathrm{~V}$, and chopping the light using an inbuilt shutter. Power calibrations were done by using a Thorlabs detector and then scaled with area.

\section{Raman characterization}

The Raman measurements were done using a "Horiba Jobin Yvon LabRAM HR Raman" instrument with the excitation wavelength of $532 \mathrm{~nm}$ using a $100 \times$ objective of numerical aperture (NA) of 0.9. Point scans were done with a laser power of approximately about $1.45 \mathrm{~mW}$ and an integration time of $8 \mathrm{~s}$.

\section{SUPPLEMENTARY MATERIAL}

Refer to the supplementary material for SEM images of AG 60 and AU 50, gate voltage dependent resistance of the AG 60 device, and comparison of Raman enhancement for AG 60, AU 50, and AG 120.

\section{ACKNOWLEDGMENTS}

The authors would like to acknowledge the usage of the facilities at the National Nanofabrication Center (NNFC, CeNSE) and the Micro/Nano Characterization Facility (MNCF, CeNSE) at IISc for the fabrication and characterization work done. The authors would also like to acknowledge the Department of Science and Technology, Government of India, for the financial support. This work was partially supported by the Ministry of Communication and Information Technology under a grant for the Centre of Excellence in Nanoelectronics, Phase II. We acknowledge funding support from MHRD, MeitY and DST Nano Mission through NNetRA. The authors are grateful to C. Miksch for the fabrication of BCML substrates. 


\section{REFERENCES}

${ }^{1}$ R. R. Nair, P. Blake, A. N. Grigorenko, K. S. Novoselov, T. J. Booth, T. Stauber, N. M. Peres, and A. K. Geim, "Fine structure constant defines visual transparency of graphene," Science 320(5881), 1308 (2008).

${ }^{2}$ A. Pradhan et al., "Ultra-high sensitivity infra-red detection and temperature effects in a graphene-tellurium nanowire binary hybrid," Nanoscale 9(27), 9284-9290 (2017)

${ }^{3}$ F. H. L. Koppens, T. Mueller, P. Avouris, A. C. Ferrari, M. S. Vitiello, and M. Polini, "Photodetectors based on graphene, other two-dimensional materials and hybrid systems," Nat. Nanotechnol. 9(10), 780-793 (2014).

${ }^{4} \mathrm{Z}$. Sun and H. Chang, "Graphene and graphene-like two-dimensional materials in photodetection: Mechanisms and methodology," ACS Nano 8(5), 4133-4156 (2014).

${ }^{5}$ G. Konstantatos et al., "Hybrid graphene-quantum dot phototransistors with ultrahigh gain," Nat. Nanotechnol. 7(6), 363-368 (2012).

${ }^{6}$ J. Fang et al., "Enhanced graphene photodetector with fractal metasurface," Nano Lett. 17(1), 57-62 (2017).

${ }^{7}$ R. Maiti, T. K. Sinha, S. Mukherjee, B. Adhikari, and S. K. Ray, "Enhanced and selective photodetection using graphene-stabilized hybrid plasmonic silver nanoparticles," Plasmonics 11(5), 1297-1304 (2016).

${ }^{8} \mathrm{U}$. J. Kim et al., "Plasmon-assisted designable multi-resonance photodetection by graphene via nanopatterning of block copolymer," ACS Photonics 2(4), 506-514 (2015).

${ }^{9}$ M. E. Ayhan, G. Kalita, M. Kondo, and M. Tanemura, "Photoresponsivity of silver nanoparticles decorated graphene-silicon Schottky junction," RSC Adv. 4(51), 26866-26871 (2014)

${ }^{10}$ S. Che, K. Jasuja, S. K. Behura, P. Nguyen, T. S. Sreeprasad, and V. Berry, "Retained carrier-mobility and enhanced plasmonic-photovoltaics of graphene via ring-centered $\eta 6$ functionalization and nanointerfacing," Nano Lett. 17(7), 4381-4389 (2017).

${ }^{11}$ Y. Yao et al., "High-responsivity mid-infrared graphene detectors with antennaenhanced photocarrier generation and collection," Nano Lett. 14(7), 3749-3754 (2014).

${ }^{12}$ Z. Fang, Z. Liu, Y. Wang, P. M. Ajayan, P. Nordlander, and N. J. Halas, "Graphene-antenna sandwich photodetector," Nano Lett. 12(7), 3808-3813 (2012).

${ }^{13} \mathrm{D}$. Paria et al., "Ultrahigh field enhancement and photoresponse in atomically separated arrays of plasmonic dimers," Adv. Mater. 27(10), 1751-1758 (2015).

${ }^{14} \mathrm{~T}$. J. Echtermeyer et al., "Strong plasmonic enhancement of photovoltage in graphene," Nat. Commun. 2(1), 458 (2011).

${ }^{15} \mathrm{Y}$. Liu et al., "Plasmon resonance enhanced multicolour photodetection by graphene,” Nat. Commun. 2(1), 579 (2011).
${ }^{16} \mathrm{D}$. Paria et al., "Graphene-silver hybrid devices for sensitive photodetection in the ultraviolet," Nanoscale 10(16), 7685-7693 (2018).

${ }^{17}$ A. G. Mark, J. G. Gibbs, T. C. Lee, and P. Fischer, "Hybrid nanocolloids with programmed three-dimensional shape and material composition," Nat. Mater. 12(9), 802-807 (2013).

${ }^{18}$ M. M. Furchi, D. K. Polyushkin, A. Pospischil, and T. Mueller, "Mechanisms of photoconductivity in atomically thin $\mathrm{MoS}_{2}$," Nano Lett. 14(11), 6165-6170 (2014).

${ }^{19}$ L. Yang, J. Deslippe, C. Park, M. L. Cohen, and S. G. Louie, "Excitonic effects on the optical response of graphene and bilayer graphene," Phys. Rev. Lett. 103, 186802 (2009).

${ }^{20}$ E. A. Taft and H. Philipp, "Optical properties of graphite," Phys. Rev. 138(1A), A197-A202 (1965).

${ }^{21}$ V. G. Kravets et al., "Spectroscopic ellipsometry of graphene and an excitonshifted van Hove peak in absorption," Phys. Rev. B 81(15), 155413 (2010).

${ }^{22} \mathrm{~K}$. Thiyagarajan, B. Saravanakumar, and S. J. Kim, "Gate-tunable photoresponse of defective graphene: From ultraviolet to visible," ACS Appl. Mater. Interfaces 7(4), 2171-2177 (2015).

${ }^{23} \mathrm{R}$. Podila et al., "Effects of disorder on the optical properties of CVD grown polycrystalline graphene," Nanoscale 4(5), 1770-1775 (2012).

${ }^{24}$ M. L. Brongersma, N. J. Halas, and P. Nordlander, "Plasmon-induced hot carrier science and technology," Nat. Nanotechnol. 10(1), 25-34 (2015).

${ }^{25}$ J. E. Lee, G. Ahn, J. Shim, Y. S. Lee, and S. Ryu, "Optical separation of mechanical strain from charge doping in graphene," Nat. Commun. 3, 1024-1028 (2012).

${ }^{26} \mathrm{G}$. Froehlicher and S. Berciaud, "Raman spectroscopy of electrochemically gated graphene transistors: Geometrical capacitance, electron-phonon, electronelectron, and electron-defect scattering," Phys. Rev. B 91(20), 205413 (2015).

${ }^{27}$ A. Pirkle et al., "The effect of chemical residues on the physical and electrical properties of chemical vapor deposited graphene transferred to $\mathrm{SiO}_{2}$," Appl. Phys. Lett. 99(12), 122108 (2011).

${ }^{28}$ Z. Fang et al., "Plasmon-induced doping of graphene," ACS Nano 6(11), 1022210228 (2012)

${ }^{29}$ G. V. Hartland, L. V. Besteiro, P. Johns, and A. O. Govorov, "What's so hot about electrons in metal nanoparticles?," ACS Energy Lett. 2(7), 1641-1653 (2017).

${ }^{30} \mathrm{M}$. Valenti et al., "Hot carrier generation and extraction of plasmonic alloy nanoparticles," ACS Photonics 4(5), 1146-1152 (2017).

${ }^{31}$ H.-H. Jeong, M. C. Adams, J.-P. Günther, M. Alarcón-Correa, I. Kim, E. Choi, C. Miksch, A. F. Mark, A. G. Mark, and P. Fischer, "Arrays of plasmonic nanoparticle dimers with defined nanogap spacers,"ACS Nano 13(10), 11453-11459 (2019).

${ }^{32} \mathrm{P}$. B. Johnson and R. W. Christy, "Optical-constants of noble-metals," Phys. Rev. B 6(12), 4370-4379 (1972). 